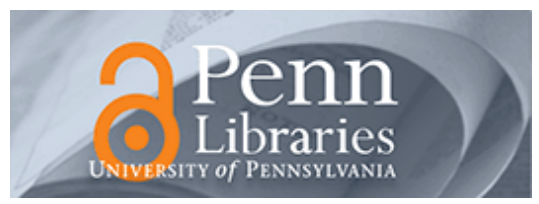

University of Pennsylvania ScholarlyCommons

April 2007

\title{
Systematic study of contact annealing: Ambipolar silicon nanowire transistor with improved performance
}

\author{
Kumhyo Byon \\ University of Pennsylvania \\ Douglas Tham \\ University of Pennsylvania \\ John E. Fischer \\ University of Pennsylvania, fischer@seas.upenn.edu
}

Alan T. Johnson

University of Pennsylvania, cjohnson@physics.upenn.edu

Follow this and additional works at: https://repository.upenn.edu/mse_papers

\section{Recommended Citation}

Byon, K., Tham, D., Fischer, J. E., \& Johnson, A. T. (2007). Systematic study of contact annealing: Ambipolar silicon nanowire transistor with improved performance. Retrieved from

https://repository.upenn.edu/mse_papers/130

Copyright (2007) American Institute of Physics. This article may be downloaded for personal use only. Any other use requires prior permission of the author and the American Institute of Physics. Reprinted in Applied Physics Letters, Volume 90, Article 143513, April 5, 2007, 3 pages.

Publisher URL: http://dx.doi.org/:10.1063/1.2720309

This paper is posted at ScholarlyCommons. https://repository.upenn.edu/mse_papers/130

For more information, please contact repository@pobox.upenn.edu. 


\title{
Systematic study of contact annealing: Ambipolar silicon nanowire transistor with improved performance
}

\begin{abstract}
High performance ambipolar silicon nanowire (SiNW) transistors were fabricated. SiNWs with uniform oxide sheath thicknesses of 6-7 nm were synthesized via a gas-flow-controlled thermal evaporation method. Field effect transistors (FETs) were fabricated using as-grown SiNWs. A two step annealing process was used to control contacts between SiNW and metal source and drain in order to enhance device performance. Initially $\rho$-channel devices exhibited ambipolar behavior after contact annealing at $400^{\circ} \mathrm{C}$. Significant increases in on/off ratio and channel mobility were also achieved by annealing.
\end{abstract}

\section{Keywords}

annealing, silicon, elemental semiconductors, nanowires, field effect transistors

\section{Comments}

Copyright (2007) American Institute of Physics. This article may be downloaded for personal use only. Any other use requires prior permission of the author and the American Institute of Physics. Reprinted in Applied Physics Letters, Volume 90, Article 143513, April 5, 2007, 3 pages.

Publisher URL: http://dx.doi.org/:10.1063/1.2720309 


\title{
Systematic study of contact annealing: Ambipolar silicon nanowire transistor with improved performance
}

\author{
K. Byon, D. Tham, and J. E. Fischer ${ }^{\mathrm{a})}$ \\ Department of Materials Science and Engineering, University of Pennsylvania, Philadelphia, \\ Pennsylvania 19104 \\ A. T. Johnson \\ Department of Physics and Astronomy, University of Pennsylvania, Philadelphia, Pennsylvania 19104
}

(Received 15 January 2007; accepted 5 March 2007; published online 5 April 2007)

\begin{abstract}
High performance ambipolar silicon nanowire (SiNW) transistors were fabricated. SiNWs with uniform oxide sheath thicknesses of 6-7 nm were synthesized via a gas-flow-controlled thermal evaporation method. Field effect transistors (FETs) were fabricated using as-grown SiNWs. A two step annealing process was used to control contacts between SiNW and metal source and drain in order to enhance device performance. Initially $p$-channel devices exhibited ambipolar behavior after contact annealing at $400{ }^{\circ} \mathrm{C}$. Significant increases in on/off ratio and channel mobility were also achieved by annealing. (C) 2007 American Institute of Physics. [DOI: 10.1063/1.2720309]
\end{abstract}

Silicon nanowires are among the most promising onedimensional nanomaterials for future nanoelectronic devices. ${ }^{1}$ Both $p$ and $n$ dopings of SiNWs can be achieved controllably, and SiNWs are compatible with existing high volume silicon manufacturing processes. ${ }^{2}$ For future applications, the control of key field effect transistor (FET) parameters such as $p$ or $n$ type, threshold voltage, on/off ratio, and channel mobility is crucial. A conventional metal-oxidesemiconductor field effect transistor (MOSFET) utilizes heavily doped silicon as source and drain and operates in inversion mode whereby a gate bias is required to generate minority carriers. ${ }^{3}$ However, most silicon nanowire field effects transistors (SiNW FETs) utilize metals as source and drain and operate in accumulation mode; a gate bias is required to generate majority carriers. It is well accepted that the characteristics of the latter are controlled by Schottky barriers between metal and conducting channels. ${ }^{4,5}$ From our previous study, we ascribed relatively low on/off ratio and channel mobility to the existence of contact barriers. ${ }^{6}$ Therefore, controlling metal/SiNW contacts is critical to achieve reliable high performance devices.

Silicide formation is one of the most effective ways to control and improve the contacts in bulk $\mathrm{Si}^{7} \mathrm{~A}$ two step annealing process is a very effective way to make reliable and low resistance nickel monosilicide (NiSi) in MOSFETs. Extensive studies focused on developing silicide formation for the $65 \mathrm{~nm}$ complementary metal-oxide-semiconductor (CMOS) technology node and beyond, and optimal processes to make self-aligned $\mathrm{NiSi}$ on top of a poly-Si gate were identified. The first annealing step between 250-350 ${ }^{\circ} \mathrm{C}$ forms $\mathrm{Ni}_{2} \mathrm{Si}$, while the second step between 400 and $450{ }^{\circ} \mathrm{C}$ is required to form low resistance NiSi in planar CMOS. ${ }^{7}$ However, only a few rigorous studies have been done to investigate the effect of contact annealing on device performance and to find the optimized contact annealing processes in SiNW systems. ${ }^{8}$

Another challenge is to align individual $p$-type and $n$-type nanowires to make large-scale complementary logic circuits. Alternatively, an ambipolar FET is very attractive

\footnotetext{
a) Author to whom correspondence should be addressed; electronic mail:
} fischer@seas.upenn.edu because it can work as either unipolar $p$-type or $n$-type FET by implementing different top gate metals and asymmetric design. ${ }^{9}$ Extensive theoretical and experimental work has been done on complementary logic devices made of ambipolar carbon nanotube transistors, ${ }^{9-11}$ while little is known about the fabrication of ambipolar FETs based on SiNWs.

We report the results of systematic annealing to control the properties of metal source and drain contacts to SiNW, and process optimization to achieve high performance ambipolar SiNW FETs.

SiNWs were synthesized via a thermal evaporation method without catalysts; details have been reported previously. ${ }^{6}$ The original growth scheme was modified to attain better control of the growth conditions. We reversed the direction of gas flow during the slow ramping-up phase of the heating schedule to ensure that minimal Si flux arrived at the substrate before the pellet reached the desired temperature, $1230{ }^{\circ} \mathrm{C}$. At this time the flow direction was reversed again to initiate growth on the collection wafer. After $30 \mathrm{~min}$ of growth the gas flow is again reversed to terminate growth at a known temperature as the furnace cools to ambient.

As-grown SiNWs were characterized using scanning electron microscopy (SEM) and transmission electron microscopy (TEM). SEM showed that most of the SiNWs were long, straight, and smooth, while TEM revealed crystalline cores covered with an amorphous oxide sheath. SiNWs grown using flow reversal had constant oxide sheath thickness of 6-7 nm regardless of the core diameter, whereas SiNWs in the previous work (without flow control) displayed a wide range of oxide sheath thicknesses of 4-12 nm. ${ }^{12}$

FETs were fabricated from SiNWs transferred onto degenerately boron-doped silicon substrates coated with $100 \mathrm{~nm}$ of stoichiometric silicon nitride $\left(\mathrm{Si}_{3} \mathrm{~N}_{4}\right)$. After locating the SiNWs with atomic force microscopy, source and drain were designed with $4 \mu \mathrm{m}$ channel length. Electron beam lithography was used to define electrodes. The SiNW oxide sheaths in the contact regions were removed with buffered HF just before e-beam evaporation of the contacts. Two consecutive layers of $10 \mathrm{~nm}$ nichrome $(\mathrm{NiCr}, 80 \% \mathrm{Ni}$ and $20 \% \mathrm{Cr}$ ) and $90 \mathrm{~nm} \mathrm{Au}$ were deposited. $\mathrm{NiCr}$ was chosen as source and drain because $\mathrm{Ni}$ provides low contact resistance and excellent compatibility with nanoscale $\mathrm{Si}^{13}$ At the same 


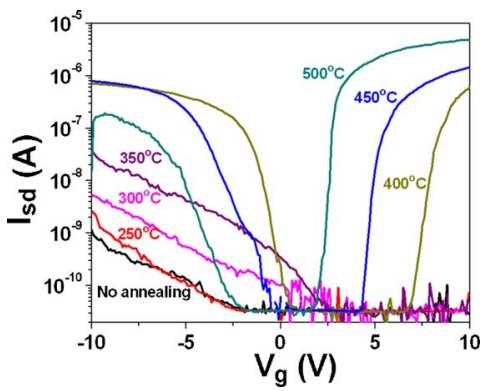

FIG. 1. (Color online) $I-V_{g}$ characteristics of a SiNW FET (device A) recorded for $V_{\text {sd }}=+2 \mathrm{~V}$ before and after contact annealing; no annealing and $T_{2}=250,300,350,400,450$, and $500{ }^{\circ} \mathrm{C}$ with fixed $T_{1}=200{ }^{\circ} \mathrm{C}$. The gate bias was swept from -10 to $+10 \mathrm{~V}$.

time, $\mathrm{Cr}$ reduces the thermal instability and poor adhesion of Ni.

Electrical transport measurements of SiNW FETs with no contact annealing were made at room temperature using the degenerately doped substrate as a back gate. After initial measurements, devices were placed in a vacuum furnace with a base pressure of $2 \times 10^{-6}$ torr. A two step annealing process was used to form reliable contact between metal and SiNW. As the first step, SiNW FET was annealed for $2 \mathrm{~min}$ at $200{ }^{\circ} \mathrm{C}\left(T_{1}\right)$. As the second step, temperature was ramped up to $T_{2}=250{ }^{\circ} \mathrm{C}$ and the sample was annealed for another $5 \mathrm{~min}$. Electrical transport measurements were carried out immediately after these two steps. After this second set of measurements, further annealing at $T_{1}=200{ }^{\circ} \mathrm{C}$ and at $T_{2}$ $=300{ }^{\circ} \mathrm{C}$ was performed and electrical transport properties again measured. This alternation between annealing and electrical measurements was continued on the same device with $T_{1}$ fixed at $200{ }^{\circ} \mathrm{C}$ and increasing $T_{2}$ in $50{ }^{\circ} \mathrm{C}$ increments up to $600{ }^{\circ} \mathrm{C}$, above which significant degradation in electrical transport properties was observed. To rule out $V_{\text {th }}$ shift from doping variations in different nanowires, we carried out contact annealing at different temperatures on the same device.

Figure 1 shows representative current versus gate voltage $\left(I-V_{g}\right)$ characteristics of device A at fixed $V_{\mathrm{sd}}=2 \mathrm{~V}$ and for second annealing temperature $T_{2}$ from 250 to $500{ }^{\circ} \mathrm{C}$. The gate sweep was from -10 to $+10 \mathrm{~V} .{ }^{14}$ Devices with unannealed contacts always behave as $p$-channel enhancement mode (normally off) FETs; at $V_{g}=0 \mathrm{~V}$, current is only tens of picoamperes (instrument limited). The $p$-type behavior is attributed to accumulation of holes in a SiNW channel, while the normally off behavior at $V_{g}=0$ is attributed to carrier depletion caused by the Schottky barriers. ${ }^{5}$ Enhancement mode is preferred for microprocessors and other logic de- vices due to faster operation and lower power consumption. ${ }^{15}$ For the first few annealing cycles, current increases and threshold voltage $\left(V_{\mathrm{th}}\right)$ becomes more positive. At $400{ }^{\circ} \mathrm{C}$ a dramatic transition to ambipolar behavior is observed; now the $I-V_{g}$ characteristic clearly shows accumulation with $V_{g}<0$, an insulating region, and an inversion region for $V_{g}>+7 \mathrm{~V}$. Such behavior requires both source and drain contacts with low barrier heights for majority and minority carriers, which can be switched between $n$ channel and $p$ channel. Therefore, the transition from unipolar $p$ channel to ambipolar is attributed to significant improvement in contacts by annealing. Beyond $550{ }^{\circ} \mathrm{C}$ we observed performance degradation, possibly due to agglomeration of the contact metal films.

We also found that annealing first at $200{ }^{\circ} \mathrm{C}$ followed by 400 or $450{ }^{\circ} \mathrm{C}$ yielded ambipolar devices with improved performance parameters. The first step is crucial to limit $\mathrm{Ni}$ diffusion and to prevent excess silicide formation. It is also effective in removing moisture and organic solvents from lift-off, since the devices were not protected by a passivation layer such as polyimide or silicon nitride. Single step annealing at $450{ }^{\circ} \mathrm{C}$ for longer time converted the SiNW into a metallic nanowire. This underscores the importance of the two-step process for reliable SiNW contacts.

Table I summarizes the parameters of device A for different second-annealing temperatures $T_{2}$. Threshold voltage $V_{\text {th }}$ is defined as the intersection of a straight line fitted to the linear region of $\log (I)$ vs $V_{g}$ and $I=30 \mathrm{pA}$. For ambipolar behavior, $V_{\text {th }}$ for holes $\left(h^{+}\right)$represents the value of $V_{g}$ at which hole conduction becomes dominant for a given $V_{\mathrm{sd}}$; similarly for electrons $\left(e^{-}\right)$it represents the onset of dominant electron conduction. The hole conduction on/off ratio is a lower limit defined as the current at $V_{g}=-10 \mathrm{~V}$ divided by the off current $\left(I_{\text {off }}\right)$, the smallest measurable current in the off state; similarly for the electron conduction on/off ratio taken at $V_{g}=+10 \mathrm{~V}$. Effective channel mobilities $(\mu)$ were estimated using $d I / d V_{g}=\mu\left(C / L^{2}\right) / V_{\text {sd }}$, where $d I / d V_{g}$ is the transconductance, $\mu$ is the carrier mobility, $L$ is the length, and $C$ is the capacitance. For this global back gate device geometry, the SiNW capacitance is given by $C \approx 2 \pi \varepsilon \varepsilon_{0} L / \ln (2 h / r)$, where $r$ is the SiNW radius, $\varepsilon$ is the gate dielectric constant, $\varepsilon_{0}$ is the permittivity of free space, and $h$ is the gate dielectric thickness. ${ }^{16}$ As well as the transition to ambipolar behavior, Table I clearly indicates significant improvement in both on/off ratio and mobilities by contact annealing.

To examine reproducibility of the annealing effects, we studied the properties of five more annealed devices (devices

TABLE I. Summary of the key parameters of a SiNW FET (device A) depending on second annealing temperatures $T_{2}$, each of which was preceded by a first anneal at $T_{1}=200{ }^{\circ} \mathrm{C}$.

\begin{tabular}{cccccccc}
\hline \hline $\begin{array}{c}\text { Annealing } \\
\text { temperature }\end{array}$ & $\begin{array}{c}\text { No } \\
\text { annealing }\end{array}$ & $250{ }^{\circ} \mathrm{C}$ & $300{ }^{\circ} \mathrm{C}$ & $350{ }^{\circ} \mathrm{C}$ & $400{ }^{\circ} \mathrm{C}$ & $450{ }^{\circ} \mathrm{C}$ & $500{ }^{\circ} \mathrm{C}$ \\
\hline$I\left(V_{g}=-10 \mathrm{~V}\right)$ & $1.2 \mathrm{nA}$ & $2.6 \mathrm{nA}$ & $5.4 \mathrm{nA}$ & $41 \mathrm{nA}$ & $700 \mathrm{nA}$ & $790 \mathrm{nA}$ & $16 \mathrm{nA}$ \\
$I\left(V_{g}=+10 \mathrm{~V}\right)$ & $28 \mathrm{pA}$ & $30 \mathrm{pA}$ & $52 \mathrm{pA}$ & $46 \mathrm{pA}$ & $600 \mathrm{nA}$ & $1.5 \mu \mathrm{A}$ & $4.8 \mu \mathrm{A}$ \\
$V_{\text {th }}$ for $h^{+}$ & $-1.8 \mathrm{~V}$ & $-2.2 \mathrm{~V}$ & $+3.0 \mathrm{~V}$ & $+2.8 \mathrm{~V}$ & $+0.3 \mathrm{~V}$ & $0.0 \mathrm{~V}$ & $-2.4 \mathrm{~V}$ \\
$V_{\text {th }}$ for $e^{-}$ & $\cdots$ & $\cdots$ & $\cdots$ & $\cdots$ & $+6.9 \mathrm{~V}$ & $+4.3 \mathrm{~V}$ & $+2.0 \mathrm{~V}$ \\
On/off ratio $\left(h^{+}\right)$ & 40 & 84 & 190 & 1900 & 26000 & 24000 & 500 \\
On/off ratio $\left(e^{-}\right)$ & $\cdots$ & $\cdots$ & $\cdots$ & $\cdots$ & 22000 & 44000 & 150000 \\
$\mu_{h}\left(\mathrm{~cm}^{2} / \mathrm{V} \mathrm{s}\right)$ & 0.1 & 0.06 & 0.51 & 2.90 & 510 & 150 & 54 \\
$\mu_{e}\left(\mathrm{~cm}^{2} / \mathrm{V} \mathrm{s}\right)$ & $\cdots$ & $\cdots$ & $\cdots$ & $\cdots$ & 310 & 730 & 770 \\
\hline \hline
\end{tabular}




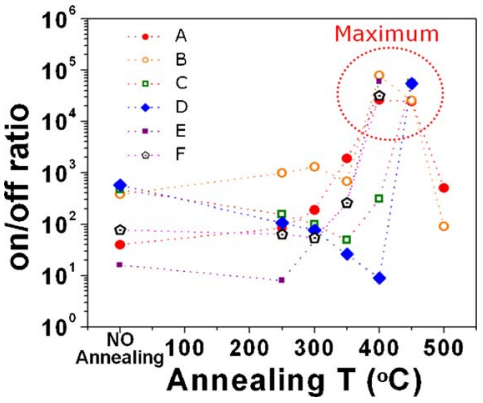

FIG. 2. (Color online) On/off ratios for hole conduction of six different devices (A-F) before and after contact annealing; no annealing and $T_{2}=250,300,350,400,450$, and $500{ }^{\circ} \mathrm{C}$ with fixed $T_{1}=200{ }^{\circ} \mathrm{C}$. Significant improvement in on/off ratio was achieved after annealing at $T_{2}=400$ and $450{ }^{\circ} \mathrm{C}$.

B-F). Figure 2 shows on/off ratios for hole conduction of all six samples as a function of $T_{2}$. Consistent improvement in on/off ratio, peaking around $400-450{ }^{\circ} \mathrm{C}$, clearly stands out from minor variations among the six samples. Contact annealing enhances the on/off ratio by two to four decades or more (limited by current noise in the experiment). Similarly, Fig. 3 shows hole and electron mobilities versus $T_{2}$. Empty circles and filled diamonds represent the effective hole and electron mobilities of each device, while the crosses are the average hole mobilities at each $T_{2}$. Significant increases were observed for both electrons and holes, and once again the enhancements were optimized at $400-450{ }^{\circ} \mathrm{C}$, coinciding with the onset of ambipolar behavior. Improvement in channel mobilities up to four orders of magnitude was achieved by contact annealing. This ambipolar behavior is attributed to accumulation of holes with negative gate bias and to inversion of electrons with positive gate bias. Two step annealing formed metal silicides with very low resistance for hole and electron conductions. The optimal annealing conditions for SiNW are very similar to those for NiSi formation in CMOS technology. ${ }^{7}$ It is very promising that the proven processes in CMOS are compatible with optimal processes for SiNWs. Further studies are required to explain the atomscale mechanisms underlying the improved device performance and ambipolar transition.

Even though tremendous progress has been made to align $p$ - and $n$-type SiNWs for logic devices, large scale complementary integrated circuits based on $p$ - and $n$-type SiNWs face huge challenges. ${ }^{1}$ In general, ambipolar behavior is not desired in CMOS because of the risk of high leakage current at the opposite polarity. However, an ambipolar

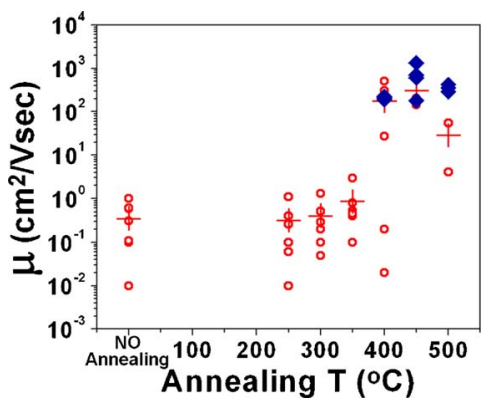

FIG. 3. (Color online) Channel mobilities for hole and electron of six different devices (A-F) before and after contact annealing; no annealing and $T_{2}=250,300,350,400,450$, and $500{ }^{\circ} \mathrm{C}$ with fixed $T_{1}=200{ }^{\circ} \mathrm{C}$. Significant improvement of mobilities and onset of ambipolar transition was achieved after annealing at $T_{2}=400$ and $450{ }^{\circ} \mathrm{C}$.
FET can perform as unipolar $p$-type or $n$-type device by engineering the top gate geometry and materials. For example, metals having different work functions can shift the threshold voltages in either direction. ${ }^{17,18}$ Therefore, large scale alternating $p$ - and $n$-type NW FETs can be made by alternating the gate metal on a very long SiNW. Recently, scientists at IBM demonstrated the first large scale logic device using ambipolar carbon nanotube FETs. They used Pd for $p$-type FET and $\mathrm{Al}$ for $n$-type FET. ${ }^{9}$ The results presented here suggest that SiNW device development will benefit directly from extensive current efforts to find the best gate metals for bulk $p$ - and $n$-channel MOSFETs. ${ }^{19,20}$

In conclusion, we achieved high performance ambipolar SiNW FETs using a two step contact annealing. Single crystalline SiNWs with uniform oxide sheath were prepared by a gas-flow-controlled thermal evaporation method. Electrical transport measurements performed on both as-prepared FETs and contact annealed FETs. An as-prepared SiNW FET behaved as a $p$-channel enhancement mode FET. After contact annealing, dramatic transition to an ambipolar FET was achieved. Ambipolar SiNW FET is one of the most promising candidates for future nanoelectronic applications. Understanding of different device physics and fabrication methods in SiNW is crucial to extend further scaling for the next generation.

The first author (K.B.) thanks Gwang-Soo Kim for valuable discussions and Scott Slavin for assistance with e-beam evaporation. This research was supported by the U.S. Department of Energy under Grant No. DE-FG02-98ER45701.

${ }^{1}$ Y. Cui and C. M. Lieber, Science 291, 5505 (2001).

${ }^{2}$ G. Zheng, W. Lu, S. Jin, and C. M. Lieber, Adv. Mater. (Weinheim, Ger.) 16, 1890 (2004)

${ }^{3}$ S. M. Sze, Physics of Semiconductor Devices, 2nd ed. (Wiley, New York, 1981).

${ }^{4}$ V. Derycke, R. Martel, J. Appenzeller, and Ph. Avouris, Appl. Phys. Lett. 80, 2773 (2002).

${ }^{5}$ F. Leonard and J. Tersoff, Phys. Rev. Lett. 84, 4693 (2000).

${ }^{6}$ K. Byon, D. Tham, A. T. Johnson, and J. E. Fischer, Appl. Phys. Lett. 87, 193104 (2005).

${ }^{7}$ L. J. Chen, Silicide Technology for Integrated Circuits (IEE, London, 2004).

${ }^{8}$ Y. Cui, Z. Zhong, D. Wang, W. U. Wang, and C. M. Lieber, Nano Lett. 3, 149 (2003)

${ }^{9}$ Z. Chen, J. Appenzeller, Y. Lin, J. Sippel-Oakley, A. G. Rinzler, J. Tang, S. J. Wind, P. M. Solomon, and Ph. Avouris, Science 311, 1735 (2006).

${ }^{10}$ M. Radosavljevic, M. Freitag, K. V. Thadani, and A. T. Johnson, Nano Lett. 2, 761 (2002).

${ }^{11}$ S. Heinze, J. Tersoff, and Ph. Avouris, Appl. Phys. Lett. 83, 5038 (2003).

${ }^{12} \mathrm{We}$ measured the diameters of $\mathrm{Si}$ crystalline core and the thicknesses of oxide sheath from energy filtered TEM images. Si core diameters range between 3 and $20 \mathrm{~nm}$, and oxide thicknesses range between 6 and $7 \mathrm{~nm}$.

${ }^{13}$ S. L. Zhang and U. Smith, J. Vac. Sci. Technol. A 22, 1361 (2004).

${ }^{14}$ Hysteresis depending on two different gate sweep directions was observed. Threshold voltage changed depending on the gate sweep directions.

${ }^{15}$ S. Datta, T. Ashley, J. Brask, L. Buckle, M. Doczy, M. Emeny, D. Hayes, K. Hilton, R. Jefferies, T. Martin, T. J. Phillips, D. Wallis, P. Wilding, and R. Chau, Tech. Dig. - Int. Electron Devices Meet. 2005, 783.

${ }^{16}$ R. Martel, T. Schmidt, H. R. Shea, and Ph. Avouris, Appl. Phys. Lett. 73, 2447 (1998).

${ }^{17}$ Y. Lin, J. Appenzeller, and Ph. Avouris, Nano Lett. 4, 947 (2004).

${ }^{18}$ J. Xiang, W. Lu, Y. Hu, Y. Wu, H. Yan, and C. M. Lieber, Nature (London) 441, 489 (2006).

${ }^{19}$ H. C. Lin, K. L. Yeh, R. G. Huang, C. Y. Lin, and T. Y. Huang, IEEE Electron Device Lett. 22, 179 (2001).

${ }^{20}$ Y. Yeo, T. King, and C. Hu, J. Appl. Phys. 92, 7266 (2002). 